\title{
Hearing Aids Reduce Daily-Life Fatigue and Increase Social Activity: A Longitudinal Study
}

Trends in Hearing

Volume 25: $1-12$

(c) The Author(s) 2021

Article reuse guidelines: sagepub.com/journals-permissions DOI: $10.1177 / 23312165211052786$ journals.sagepub.com/home/tia

(S)SAGE

\author{
Jack A. Holman' (D), Avril Drummond², and Graham Naylor'
}

\begin{abstract}
People with hearing loss experience fatigue, and it is unknown whether this is alleviated by treatment with hearing aids. The objective of this study was to address this issue and to investigate the possible concomitant effect of hearing-aid fitting on activity levels. An intervention group $(n=53)$ who were due to be fitted with their first-ever hearing aid(s) and a control group $(n=53)$ who had hearing loss but no change in hearing aid status-completed a battery of self-report outcome measures four times: once before fitting, and at 2 weeks, 3 months, and 6 months post fitting. Self-report outcome measures at each assessment captured fatigue, listening effort, hearing handicap, auditory lifestyle, social participation restrictions, and work, social and physical activity levels. Hearing-aid fitting led to a significant reduction in listening-related fatigue, but not general fatigue, in the intervention group compared to the control group. Additionally, social activity level increased and social participation restriction decreased significantly after hearing aid fitting in the intervention group compared to the control group. No significant interaction was found between working status and change in listening-related fatigue score. This study is the first to make a longitudinal measurement of fatigue before and after first-ever hearing aid fitting and to identify an increase in social activity level after hearing aid fitting. These findings have important implications for future research and the clinical practice of hearing aid fitting.
\end{abstract}

\section{Keywords}

hearing loss, hearing handicap, listening effort, listening fatigue

Received II May 202I; accepted 3I July 202 I

\section{Introduction}

Hearing loss can affect people's lives beyond their difficulty hearing (Heffernan et al., 2016). One effect which has attracted increased interest in recent years is the potential additional fatigue experienced in everyday life by people with hearing loss. While the experience of having fatigue may in itself be negative, fatigue can also negatively impact other health-related outcomes such as activity and quality of life (Flensner et al., 2013). Thus, it is important to investigate ways of limiting the development and impact of fatigue in this population.

The basic and most intuitive theory of listening-related fatigue states that the cognitive effort required in challenging listening situations constitutes a drain on finite cognitive resources, resulting in fatigue (Hornsby et al., 2016). However, factors such as motivation play an important role, meaning that the development of listening-related fatigue is multi-faceted (Hockey, 2013). While listening effort does seem to be the main instigator of fatigue for people with a hearing loss (who experience challenging listening relatively frequently), the development of fatigue in some has also been linked to the negative emotions associated with having a hearing loss (Holman et al., 2019). A systematic review found some evidence that people with hearing loss experience greater fatigue than those without (Holman et al., 2021a). However, not all research has identified increased levels of fatigue in those with hearing loss. Using Ecological Momentary Assessment, Burke and Naylor (2020) found no difference in general daily-life fatigue between people with hearing losses, as determined by audiometry, and those

\footnotetext{
'Hearing Sciences (Scottish Section), Mental Health and Clinical Neurosciences, School of Medicine, University of Nottingham, Glasgow, UK ${ }^{2}$ School of Health Sciences, University of Nottingham, Nottingham, UK

\section{Corresponding Author:}

Jack A. Holman, Hearing Sciences (Scottish Section), Mental Health and Clinical Neurosciences, School of Medicine, University of Nottingham, Level 3 New Lister Building, Glasgow Royal Infirmary, 16 Alexandra Parade, Glasgow, G3I 2ER, UK.

Email: jack.holman@nottingham.ac.uk
} 
without. However, the control group in that study did have hearing difficulties such as tinnitus.

Logically, an increase in audibility through the fitting of a hearing device should reduce the listening effort required in any given situation, and in turn reduce fatigue. Evidence regarding the impact of hearing aid fitting (as opposed to cochlear implant fitting) on fatigue has focused to date mainly on the cross-sectional study of people wearing and not wearing hearing devices (Alhanbali et al., 2017; Bisgaard \& Ruf, 2017). Such research has provided mixed evidence and, by virtue of using self-report questionnaires, invariably measures long-term fatigue. However, Hornsby (2013) used a crossover study design to measure transient fatigue and listening effort and identified an objective benefit of hearing aid fitting through better performance using a dual-task paradigm. The lack of subjective benefit found by that study suggests that subjective and objective measures of transient fatigue may not tap into the same phenomenon. Regarding long-term fatigue, it is possible that general fatigue questionnaires may not consistently detect any beneficial impact of hearing device fitting on fatigue, due to a lack of sensitivity.

Listening-related fatigue affects people in different ways, and may also be managed differently from person to person (Davis et al., 2021; Holman et al., 2019). As listening-related fatigue is generally contingent on engagement in conversational activity, it has been postulated that activity levels (social, work, and physical) and listening-related fatigue are related and may affect well-being (Holman et al., $2021 b)$. It is thus possible that more conversationally active lifestyles, such as being involved in workplace environments, would lead to greater instances of listening-related fatigue. Additionally, there is cross-sectional evidence that hearing aid use is linked to increased social activity levels (Fisher et al., 2015; Lee \& Noh, 2015; Sawyer et al., 2019). Therefore, the impact of hearing aid fitting on listening-related fatigue may be connected to daily-life activity, and the impact of hearing aid fitting on well-being would be dependent on what, if any, changes there were in both fatigue and activity. Examining interactions between fatigue and activity has not been a feature of quantitative research in the field, which may account for discrepancies between studies. Other individual differences may have a role in listening-related fatigue: Perceived hearing difficulty rather than audiometrically measured hearing loss has previously been linked to subjective fatigue (Hornsby \& Kipp, 2016). Additionally, one's inclination towards effortful cognitive activity (need for cognition) has been identified as related to the amount of effort exerted in group situations (Smith et al., 2001). This could be important when it comes to the development of listening-related fatigue, particularly given the important role of motivation in fatigue (Hockey, 2013). Age and gender are not related to most outcomes of hearing aid fitting (Vestergaard Knudsen et al., 2010). However, as relatively little is known regarding listening-related fatigue, age and gender should be controlled for in studies.
Longitudinal assessment of fatigue before and after hearing aid fitting has not previously been reported. Therefore, we conducted a longitudinal study to address the following research questions:

(Q1) Does first-ever hearing aid fitting have an impact on fatigue?

We hypothesized that fatigue would reduce post-hearing aid fitting.

(Q2) What variables may influence the impact of hearing aid fitting on fatigue?

Demographic, hearing, and lifestyle factors could be associated with the impact of hearing aid fitting on fatigue. It was hypothesized that age, gender, hearing loss, hearing handicap, social activity level, work activity, and need for cognition (inclination towards effortful cognitive activity) would be associated with fatigue scores. It was hypothesized that listening effort, being functionally linked to fatigue, would correlate temporally with fatigue.

(Q3) Do levels of social or physical activity change after hearing aid fitting, and is this related to fatigue?

We hypothesized that social and physical activity would rise after hearing aid fitting. Associated variables of interest for social activity were age, gender, hearing handicap, auditory lifestyle demand, and need for cognition. Associated variables of interest for physical activity were age, gender, and hearing handicap. We hypothesized that social and physical activity would rise for participants who experienced a reduction in fatigue.

\section{Materials and Methods}

This research received ethical approval from the West of Scotland Research Ethics Committee (18/WS/0030) and the NHS R\&D (GN18EN073).

\section{Design}

Fatigue was measured before, and at three timepoints after first-ever hearing aid fitting in an intervention group. A control group of people with hearing loss, some with and some without hearing aids, also completed the same measurement regime (Figure 1). Additionally, several variables potentially related to fatigue were measured to assess potential mediating or associated factors, including accounting for individual differences at assessment 1 (baseline).

\section{Participants and Recruitment}

Participants were between 18 and 75 years old. For inclusion in the intervention group participants had to have self- 


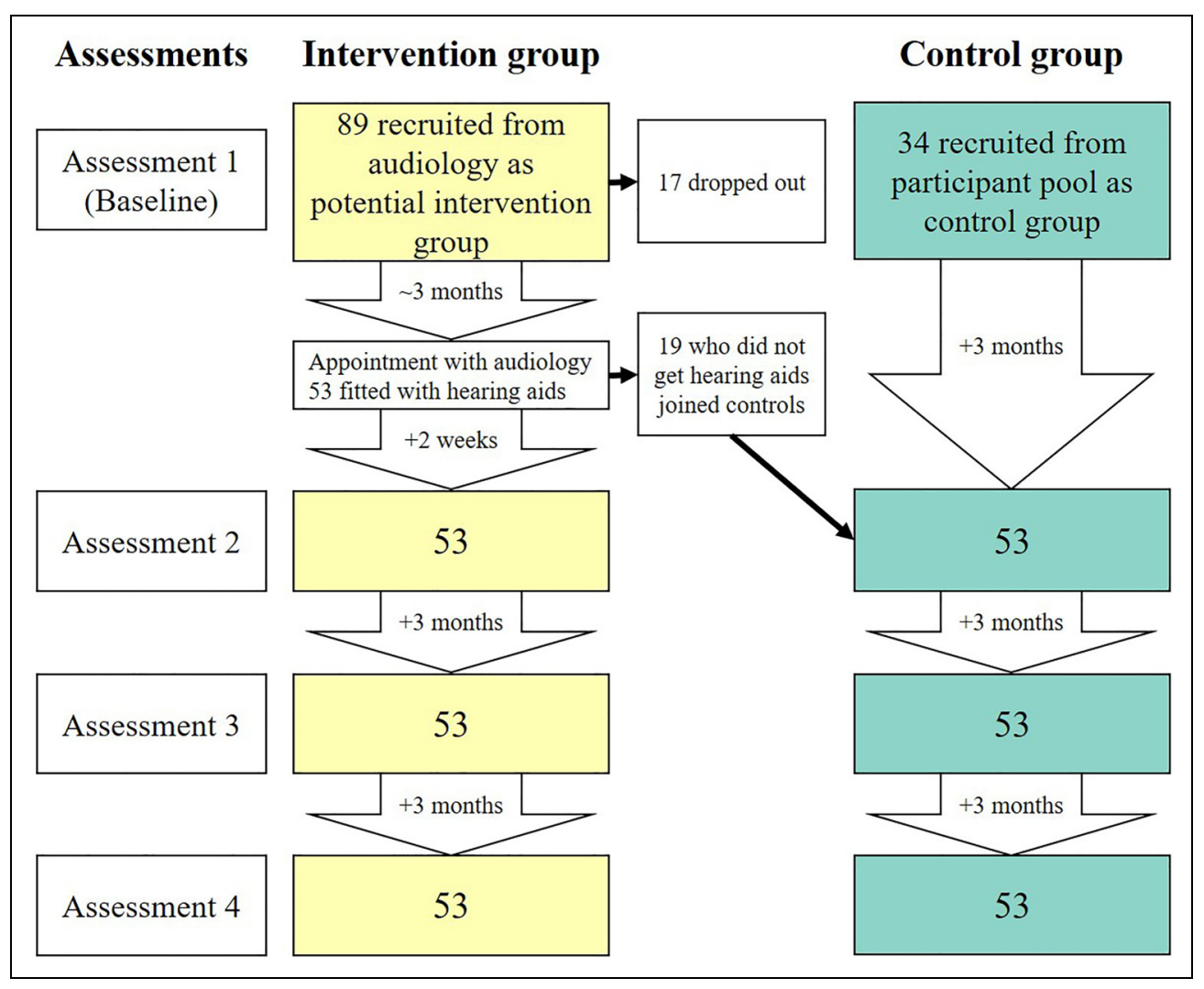

Figure I. Timeline of assessments and participant numbers for control and intervention groups. The assessments are displayed on the left of the figure, with the timing of assessments displayed in downward arrows for each group. Dropouts occurred throughout the course of the study but are listed at baseline.

reported hearing difficulties, be receiving their first-ever hearing aid(s), and not be attending the audiology clinic with a primary complaint of tinnitus or vestibular issues. For inclusion in the control group participants had to have self-reported hearing difficulties and have experienced no

Table I. Group Hearing and Demographic Characteristics.

\begin{tabular}{|c|c|c|}
\hline$N$ & $\begin{array}{l}\text { Intervention } \\
53\end{array}$ & $\begin{array}{l}\text { Control } \\
53\end{array}$ \\
\hline Female & 20 & 29 \\
\hline Age range (mean, $S D)$ & $46-75(61.4,7.6)$ & 20-73 (66, II.9) \\
\hline $\begin{array}{l}\text { BE4FA range (mean, } \\
\text { SD) }\end{array}$ & $\begin{array}{l}10-88.75(32.3 \\
\text { 13) }\end{array}$ & $\begin{array}{l}\text { 7.5-107.5 (35.8, } \\
19.9)\end{array}$ \\
\hline $\begin{array}{l}\text { WE4FA range (mean, } \\
\text { SD) }\end{array}$ & $\begin{array}{l}22.5-90(40.1 \\
14.5)\end{array}$ & $15-110(44.4,23.5)$ \\
\hline 0 hearing aids & 0 & 29 \\
\hline I hearing aid & 28 & 10 \\
\hline 2 hearing aids & 25 & 14 \\
\hline Tinnitus & 18 & 24 \\
\hline Employed & 14 & 12 \\
\hline
\end{tabular}

Abbreviations: BE4FA = better ear four frequency average hearing loss $(\mathrm{dBHL}) ; \mathrm{WE} 4 \mathrm{FA}=$ worse ear four frequency average hearing loss ( $\mathrm{dBHL}) ; \mathrm{SD}$ $=$ standard deviation. change in hearing aid status for at least 1 year (i.e., hearing aid users not stopping or getting new hearing aids, and nonusers not getting a first hearing aid). Though not individually matched, the control group was kept as close as possible to the intervention group for gender, mean age, and mean fourfrequency average hearing loss (see Table 1).

Participants for the intervention group were recruited from the waiting list for audiological evaluation at the Glasgow Royal Infirmary audiology department, except for two participants recruited through word of mouth. The first (baseline) assessment was conducted before each participant's audiological screening, and for this reason, not all potential intervention group participants ended up receiving hearing aids. Those who were not fitted with a hearing aid became part of the control group. The remaining participants in the control group were selected from the Hearing SciencesScottish Section participant database. The stability of hearing aid status (i.e., that participants have not started or stopped using hearing aids) was checked at the start of each assessment to ensure participants remained eligible for their designated group.

A minimum sample size of 90 participants ( 45 per group) was estimated based on a between-group analysis of covariance (ANCOVA), with a power of $80 \%(\beta=0.2)$, capable of 
detecting an effect size of 0.3 and a type one error rate alpha of 0.05 . The power calculation was conducted using $\mathrm{G}^{*}$ power calculator version 3.1. The sample size calculation was based on the Fatigue Assessment Scale (FAS) as this was the questionnaire used in the most relevant previous research (Alhanbali et al., 2017).

In total 129 participants completed at least one assessment, and 106 (49 female) completed all four assessments. Altogether 89 participants were recruited for potential inclusion in the intervention group. Seventeen of those participants did not complete all four assessments, dropping out for reasons such as personal circumstances, failure to respond to communications, and failure to meet the inclusion criteria that were not initially detected. Seven participants were offered a hearing aid at their clinical appointment, but chose not to be fitted, and 12 were told that a hearing aid would not be appropriate. Table 1 shows the hearing and demographic characteristics of participants. Four participants from the control group and 10 from the intervention group had worse-ear four-frequency averages (WE4FA) of < $25 \mathrm{~dB} \mathrm{HL}(500 \mathrm{~Hz}, 1,2$, and $4 \mathrm{kHz})$, which in some circumstances is categorized as no hearing loss. However, fourfrequency averages do not always highlight significant losses at individual frequencies which could be a cause of hearing loss necessitating hearing amplification. All participants had self-reported hearing difficulties based on a 5-point scale from no hearing loss to profound hearing loss.

\section{Procedure}

Data collection ran from March 2018 until December 2019. Each participant made four visits to the department. For the intervention group there was one assessment before hearing aid fitting, and then 2 weeks, 3 months, and 6 months post fitting (equating to 3 months between each assessment). These time points were chosen as the first few weeks after a hearing aid fitting involve potentially troublesome sensory and psychosocial adjustments (Dawes et al., 2014), whereas by 3 months post-fitting substantial performance improvements are regularly shown (Munro, 2008; Munro \& Lutman, 2003). The only time interval that varied between participants in the intervention group was from assessment 1 (baseline) to assessment 2, with some being 3 months apart and others being longer, depending on the availability of audiological screening appointments. The assessments for the control group were each spaced 3 months apart. The timeline of sessions and participant numbers is shown in Figure 1.

During assessment 1 , which took $\sim 1 \mathrm{~h}$, participants provided written informed consent and were then asked about their hearing history (including hearing aid use and tinnitus), underwent ear examination and audiometric assessment, and completed research questionnaires. At subsequent assessments, participants completed the same questionnaires apart from the "Need for Cognition Scale," and there was no repeat of the pure tone audiometry or history taking. Continued hearing aid use (or non-use for certain members of the control group) was ascertained at each assessment by asking how many hearing aids they own and how often they wear them (never, sometimes, always). While some participants rarely used their new hearing aids, no participants gave up on their hearing aids entirely. At the final assessment, participants were debriefed and offered the opportunity to ask additional questions about the study. Appointments were arranged by either phone or email, depending on the participant's preference, and appointment reminders were also provided in the same way. Participants received $£ 50$ compensation in total; $£ 10$ at each of the first three visits, and $£ 20$ at the final visit.

\section{Outcome Measures}

The outcome measures used in the study were 13 self-report questionnaires which addressed fatigue, listening effort, hearing handicap, activity (social work and physical), social participation restrictions, auditory lifestyle, and need for cognition. For the purposes of this study, participants were asked to respond with respect to their current level of hearing aid usage (i.e., in situations where they would be wearing their hearing aids, answering the corresponding question as though wearing hearing aids). Full questionnaire details are provided in Supplemental Digital Content 1.

Three self-report questionnaires in total were utilized to measure fatigue. Two of the questionnaires assessed long-term fatigue. The FAS (Michielsen et al., 2003) is a widely used unidimensional general fatigue scale. The multidimensional fatigue symptom inventory (MFSI) (Stein et al., 2004) measures the domains of general fatigue, physical fatigue, emotional fatigue, mental fatigue, and vigor (energy, active force, or power ["Vigour, 2020"]). One self-report questionnaire assessed listening-related fatigue specifically. The 40-item Vanderbilt Fatigue Scale for Adults (VFS-A-40) (Hornsby et al., 2021) comprises both unidimensional and multidimensional scales (cognitive fatigue, emotional fatigue, physical fatigue, and social fatigue).

Listening effort was assessed using the Listening Effort Assessment Questionnaire (EAS) (Alhanbali et al., 2017) which measures the amount of effort participants use "listening in everyday life." Hearing handicap was assessed using the Hearing Handicap Inventory for the Elderly/Adults (HHIE/A) (Newman et al., 1990; Ventry \& Weinstein, 1982), the HHIE for people aged 65 years and over; otherwise HHIA.

Social activity level was assessed using two self-report questionnaires. The social activity $\log$ (SAL) (Syrjala et al., 2010) measures the quantity of social activity in the "past week" and "past month." The Social Participation Questionnaire (SPQ) (Densley et al., 2013) measures the quantity of social activity undertaken in the "last twelve months." Work activity was assessed using the "how do 
you spend your time?" section of the Craig Handicap Assessment and Reporting Technique (CHART) (Whiteneck et al., 1992). Physical activity in the "last seven days" was assessed using the International Physical Activity Questionnaire (IPAQ) (Craig et al., 2003).

The experiential component of social activity was assessed using the Social Participation Restrictions Questionnaire (SPaRQ) (Heffernan et al., 2018; Heffernan et al., 2019) which consists of two scales assessing "social behaviours" and "social perceptions." Auditory lifestyle was assessed using the Auditory Lifestyle and Demand Questionnaire (ALDQ) (Gatehouse et al., 1999). The tendency for an individual to engage in and enjoy thinking was assessed using the Need for Cognition Scale (NFC) (Cacioppo \& Petty, 1982).

\section{Statistical Analysis}

Statistical analyses were carried out using $\mathrm{R}$ version 3.6.1 (R Core Team, 2019). For analyses of group means, analyses of covariance (ANCOVA) were conducted where appropriate. As the data were longitudinal and the individual trajectories were important, multilevel modeling (growth curve modeling specifically) was used as the primary approach to analysis. This was analyzed using the nlme package (Pinheiro et al., 2019). The hierarchy in the data set was repeated measures (level 1) nested within individual participants (level 2).

Associations between-group (intervention vs. control) and outcome measure scores at baseline were assessed using independent-sample $t$-tests, or Mann-Whitney $U$-tests. Group mean differences at each subsequent time point, controlling for baseline score, were analyzed using ANCOVA or nonparametric equivalent. The sm package was used to analyze non-parametric ANCOVA (Bowman \& Azzalini, 2018). Separate multilevel growth models were built to assess the relationships between group and outcome measures across time for individuals, followed by assessment of the intervention group alone where relevant. Kendall rank correlation coefficients $(r \tau)$ were used to assess the relationships between the magnitude of change in relevant variables.

\section{Results}

In all following text, the pre-fitting assessment is termed "baseline" with the post-fitting assessments denoted as 2, 3, and 4. A Spearman rank correlation matrix of all baseline questionnaires is provided in Supplemental Digital Content 2.

\section{Research Question I: Does First-Ever Hearing Aid Fitting Have an Impact on Fatigue?}

On the basis of ANCOVA analysis controlling for baseline scores, FAS scores at assessment 2 differed significantly between groups $(H=2.82, p=.012)$, but this was not the case at assessments 3 or 4 (Figure 2). The significant result at assessment 2 seems to be anomalous, driven by changes in both groups from baseline in the opposite direction to that seen in assessments 3 and 4 . There was no significant difference between groups at any of the three post-fitting assessments for total MFSI score after controlling for individual participants' baseline scores on the outcome measure in question (Figure 2). Unidimensional VFS-A-40 scores, after controlling for individual participants' baseline scores on the outcome measure in question, were significantly lower (better) in the intervention group at all subsequent assessments $(2: F[1,103]=16.33, p=<.001 ; 3: F[1,103]=40$, $p=<.001 ; 4: F[1,103]=39.8, p=<.001$ ) (Figure 2). Effect sizes based on adjusted means at each assessment were $d=0.506, d=0.75$, and $d=0.79$.

Analysis of the multidimensional scales of the MFSI, after controlling for individual participants' baseline scores on the outcome measure in question, found no significant differences between groups in any assessment for physical fatigue, mental fatigue, or emotional fatigue. The vigor scale of the MFSI showed that after controlling for individual participants' baseline scores on the outcome measure in question, intervention group scores were significantly higher (better) than the control group at the final two assessments, $(F[1,103]=4.56, p=.039 ; F[1,103]=6.19, p=.015)$. VFS-A-40 scores (baseline corrected) for cognitive fatigue, emotional fatigue, and social fatigue were lower in the intervention group than the control group at assessment $2,(F[1$, $103]=19.01, p=<.001 ; F[1,103]=11.25, p=.001 ; F[1$, $103]=13.74, p=<.001)$, assessment $3,(F[1,103]=41.12$, $p=<.001 ; F[1,103]=22.25, p=<.001 ; F[1,103]=36.69$, $p=<.001)$, and assessment $4,(F[1,103]=32.86, p=$ $<.001 ; F[1,103]=35.67, p=<.001 ; F[1,103]=49.91, p=$ $<.001)$. There was no group difference for physical fatigue. Based on these results, there does appear to be a beneficial impact of first-ever hearing aid fitting on fatigue (Q1).

Following on from the group-level analysis, multilevel growth model analysis was conducted to assess fatigue at the participant level across both groups. Group and baseline HHIE/A scores were significantly associated with VFS-A-40 score, with fatigue increasing with HHIE/A score (Table 2). Assessment number was not significantly associated with VFS-A-40 score, but the interaction between the group and assessment number was, suggesting that the difference between groups varied across assessments. The group difference in VFS-A-40 score increased by 1.2 for each assessment over time. There was no significant association between the VFS-A-40 score and age, sex, BE4FA, WE4FA, NFC score, or baseline SAL score.

\section{Research Question 2: What Variables may Influence the Impact of Hearing Aid Fitting on Fatigue?}

To further investigate the impact of hearing aid fitting on fatigue by breaking down the interaction term (group $\times$ 


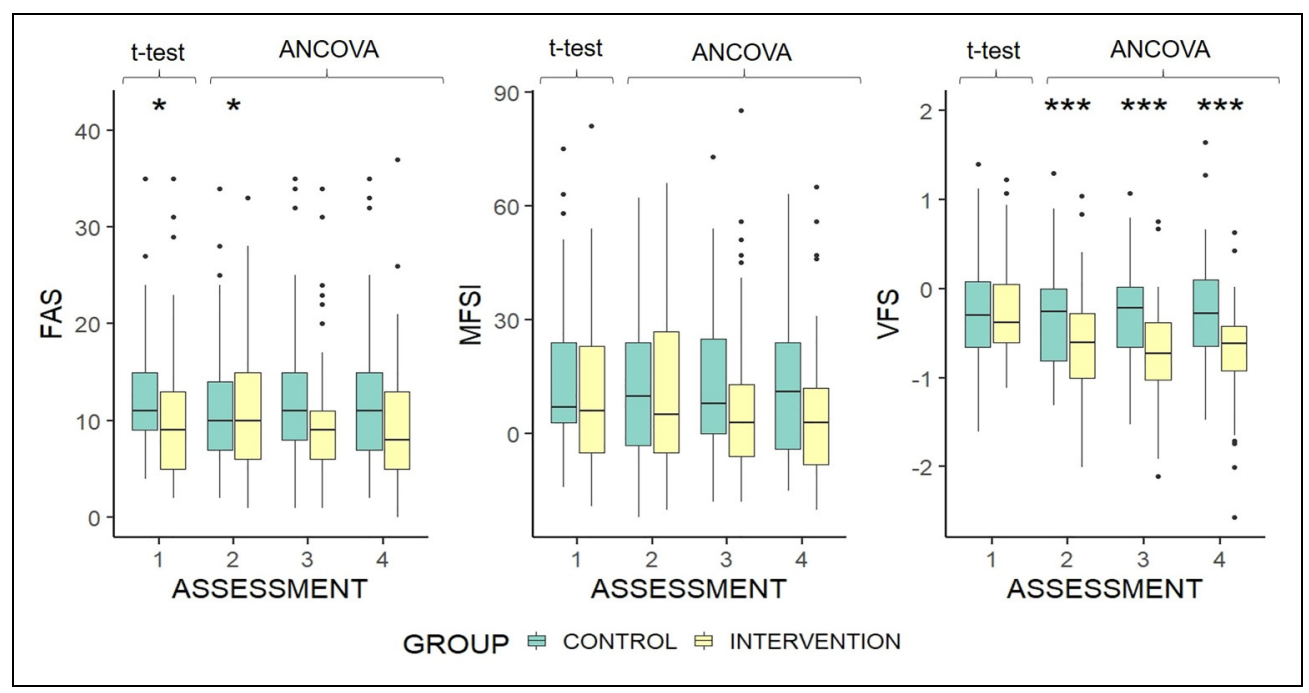

Figure 2. Boxplots of fatigue assessment scale, multidimensional fatigue symptom inventory, and the 40 -item Vanderbilt fatigue scale for adults score by group and assessment. Group difference at baseline is based on independent samples t-test. Differences at each subsequent time point are based on ANCOVA, controlled for baseline scores. Higher scores indicate greater fatigue. $*_{p}<.05 * * p<.01 * * * p<.001$. The upper and lower hinges represent 25th and 75th percentiles, respectively. The upper whisker is the highest value within the 1.5 Xinter-quartile range, measured from the upper quartile. The lower whisker is the lowest value within the $1.5 \times$ interquartile range, measured from the lower quartile. Values beyond this are represented as outlier dots.

assessment) and re-assessing the relationship between fatigue and associated variables, a new multilevel growth model was created for the intervention group only (Table 3 ). The slopes did not vary significantly across participants, $S D=0.46(95 \%$ CI: $0.36,0.6), x^{2}(2)=3.92, p=.14$, suggesting that participants in the intervention group tended to follow the same trend across time. A logarithmic function of time was found to be a better fit than linear, quadratic, and cubic, $S D$ $=0.4(95 \%$ CI: $0.31,0.5), x^{2}(2)=4.66, p=.03$.

Although the growth model analysis suggested a logarithmic trend of VFS-A-40 over time, visualization of the

Table 2. Effects of Predictors on Vanderbilt Fatigue Scale for Adults Score: all Participants.

\begin{tabular}{lclc}
\hline Construct & B & SE & $T$ \\
\hline Group & -0.25 & 0.09 & $-2.88^{* *}$ \\
Assessment number & -0.46 & 0.36 & 1.28 \\
Age (GMC) & -0.004 & 0.004 & -0.9 \\
Sex & 0.13 & 0.082 & 1.59 \\
BE4FA & -0.001 & 0.004 & -0.29 \\
WE4FA & 0.002 & 0.004 & 0.53 \\
Baseline SAL & 0.008 & 0.039 & 0.21 \\
Baseline HHIE/A & 0.016 & 0.002 & $7.28^{* * *}$ \\
NFC & 0.001 & 0.003 & 7.28 \\
Group $\times$ Assessment & 1.2 & 0.51 & $2.37^{*}$ \\
\hline
\end{tabular}

Abbreviations: $B=$ unstandardized beta; $\mathrm{SE}=$ standard error; $t=$ test statistic; $G M C=$ grand mean centered; $S A L=$ social activity log; $H H I E / A=$ hearing handicap inventory for the elderly/adults; NFC = need for cognition scale.

$*_{p}<.05 * * p<.01 * * *_{p}<.001$. individual VFS-A-40 trajectories highlights that individuals had a wide variety of score trajectories from baseline to assessment 4 (Supplemental Digital Content 2).

Baseline HHIE/A was significantly and positively associated with VFS-A-40 score in the intervention group, and assessment number was significantly and negatively associated with VFS-A-40 score (Table 3). There was a decrease in score of -0.35 for each additional assessment over time. There was no significant association between the VFS-A-40 score and age, sex, BE4FA, WE4FA, NFC score, or baseline SAL score.

After controlling for individual participants' baseline scores on the outcome measure in question, listening effort

Table 3. Effects of Predictors on Vanderbilt Fatigue Scale for Adults Score: Intervention Group.

\begin{tabular}{lllc}
\hline Construct & $B$ & SE & $T$ \\
\hline Assessment number & -0.35 & 0.044 & $1.74^{* * * *}$ \\
Age (GMC) & -0.003 & 0.009 & -0.29 \\
Sex & 0.16 & 0.13 & 1.26 \\
BE4FA & -0.003 & 0.007 & -0.45 \\
WE4FA & 0.0002 & 0.006 & 0.03 \\
Baseline SAL & 0.002 & 0.065 & 0.24 \\
Baseline HHIE/A & 0.012 & 0.003 & $3.4^{* *}$ \\
NFC & -0.007 & 0.005 & -1.5 \\
\hline
\end{tabular}

Abbreviations: $B=$ unstandardized beta; $S E=$ standard error; $t=$ test statistic; $\mathrm{SAL}=$ social activity log; $\mathrm{GMC}=$ grand mean centered; $\mathrm{HHIE} / \mathrm{A}=$ hearing handicap inventory for the elderly/adults; NFC $=$ need for cognition scale.

$*_{p}<.05 * * p<.01 * * * p<.001$. 
significantly decreased in the intervention group post-fitting compared to the control group (EAS: $F[1,103]=11.04, p$ $=.0012 ; F[1,103]=32.92, p=<.0001 ; F[1,103]=22.46$, $p=<.0001)$. Effect sizes based on adjusted means at each assessment were $d=0.5, d=0.82$, and $d=0.66$. In the intervention group, the change in VFS-A-40 score from baseline was significantly correlated with the change in EAS score at all follow-up assessments (2: $r \tau=0.32, p=.0007 ; 3: r \tau=$ $0.22, p=.02 ; 4: r \tau=0.42, p=<.0001)$.

Work Activity. Mean VFS-A-40 scores for intervention group participants who work were higher at each assessment than for those who were not in work. However, this difference was not statistically significant at baseline, $t=-0.57, p=$ .57. There was a significant difference when controlling for baseline scores at the second assessment, $(F[1,50]=6.52$, $p=.014)$, but not at the final two post-intervention assessments, $(F[1,50]=0.62, p=.44 ; F[1,50]=3.9, p=.054)$. The lack of a consistent pattern suggests that postintervention VFS-A-40 scores changed at a similar rate in both working and non-working participants.

In summary, hearing handicap at baseline is the only prefitting variable which was significantly associated with a change in fatigue, and which therefore may influence the impact of hearing aid fitting on fatigue (Q2).

\section{Research Question 3: Do Levels of Social or Physical Activity Change after Hearing Aid Fitting, and is This Related to Fatigue?}

To answer this compound question, we analyzed the activity levels across time between the intervention and control groups, followed by a correlation analysis of the temporal changes in activity and fatigue.

Social Activity. ANCOVA analysis found that SAL scores, controlling for baseline score, were significantly higher in the intervention group than the control group at assessments 2, 3, and 4 (Figure 3). However, the mean SAL score for the control group dropped from baseline to assessment 2, which may have exaggerated the effect of hearing aid fitting. SPQ scores were significantly higher in the intervention group at the final assessment, with control group scores steady across assessments (Figure 3). Effect sizes based on adjusted means at each assessment were $d=0.36, d=0.58$, and $d=$ 0.52 .

Group, assessment number, baseline ALDQ, and NFC were significantly and positively associated with SAL score (Table 4). Baseline HHIE/A was significantly and negatively associated with the SAL score. Increases of one unit in baseline HHIE/A score were related to a decrease in SAL score of 0.012. There was no significant association between age, sex, or the interaction of group and assessment and SAL score.
As group was significantly associated with SAL score, further analysis was conducted on the intervention group alone. For this group, assessment and baseline ALDQ score were significantly and positively associated with SAL score (Table 5). Baseline HHIE/A score was significantly and negatively associated with SAL score. Increases of one unit in baseline HHIE/A score were related to a decrease in SAL score of 0.02 . There was no significant association between SAL score in the intervention group and age, sex, or NFC.

Given that social activity increased significantly after hearing aid fitting, the change in social participation restriction was also investigated. It was found that the social behavior subscale of the SPARQ reduced (improved) significantly more in the intervention group than the control group when controlling for baseline scores at each assessment after hearing aid fitting, $(F[1,103]=8.2, p=.005 ; F[1,103]=$ $21.5, p=<.0001 ; F[1,103]=17.26, p=<.0001)$. The social perception subscale also reduced (improved) significantly in the intervention group at each post-intervention assessment, $(F[1,103]=4.16, p=.044 ; F[1,103]=25.89$, $p=<.0001 ; F[1,103]=19.35, p=<.0001)$.

For participants in both groups, the changes in VFS-A-40 and SAL scores from baseline to assessments 2 and 3 were not significantly correlated, but at assessment 4 the changes from baseline were significantly correlated $(r \tau=-0.17, p=$ .009). However, when the intervention group alone was analyzed, the correlation became marginal $(r \tau=-0.18, p=$ .064). Therefore, social activity levels increased with hearing aid fitting, but there is no evidence that decreasing fatigue is associated with increasing social activity (Q3).

Physical Activity. There was no mean difference in IPAQ scores between groups at baseline, $U=1,383, p=.89$. There was also no significant difference when controlling for baseline scores at any of the post-intervention assessments, $F(1,103)=0.45, p=0.5 ; F(1,103)=0.58, p=.45$; $F(1,103)=1.05, p=.31$. Therefore, there is no evidence of physical activity being affected by hearing aid fitting.

\section{Discussion}

This study was designed to examine the impact of hearing aid fitting on fatigue, and variables related to changes in fatigue (research question Q1), whether activity changed after hearing aid fitting (Q2), and whether this had any link to fatigue (Q3). The two general fatigue questionnaires (FAS and MFSI) showed no difference between groups postfitting. This finding supports the results of Alhanbali et al. (2017), where FAS scores were not significantly different for hearing loss groups with or without hearing aids. While cross-sectional research using another long-term general fatigue questionnaire has previously found a significant effect (Bisgaard \& Ruf, 2017), the majority of support for a beneficial impact of hearing device fitting on long-term general fatigue has come from prospective non-randomized 


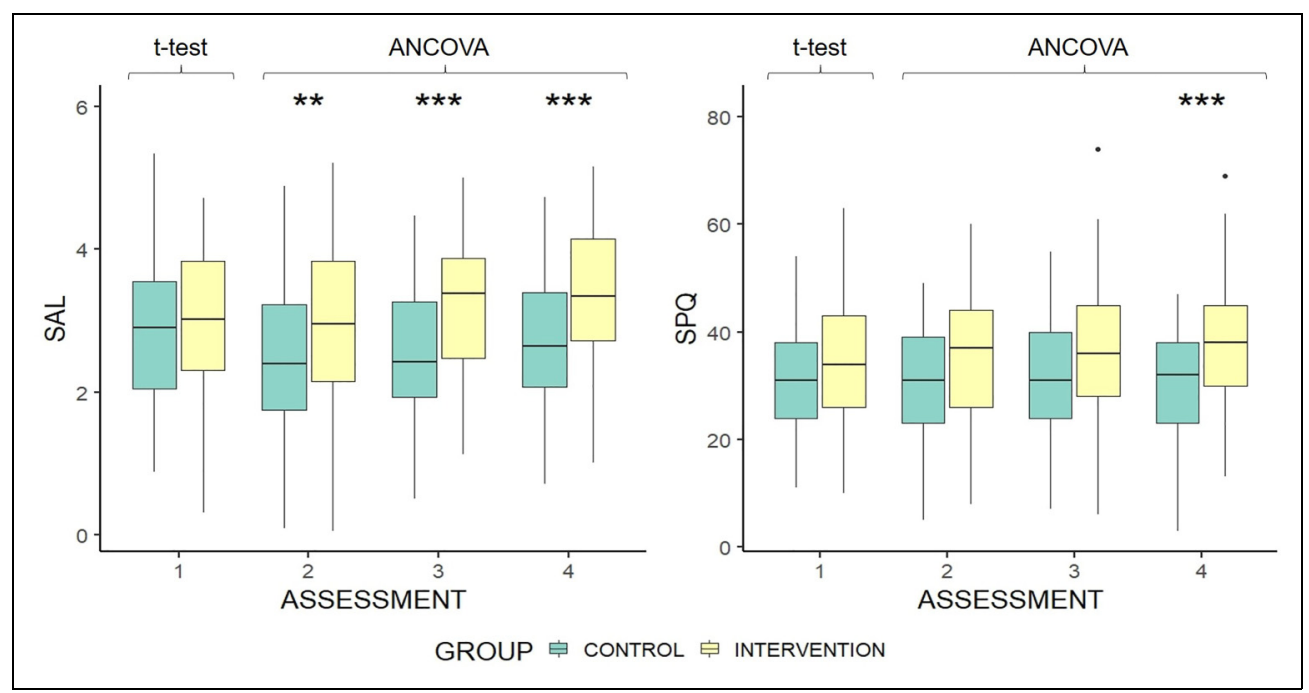

Figure 3. Boxplots of social activity log \& social participation questionnaire score by group and assessment. Group difference at baseline is based on independent samples $t$-test. Differences at each subsequent time point are controlled for baseline scores. Higher scores indicate greater social activity. $*_{p}<.05 * * p<.01 * * * p<.001$. The upper and lower hinges represent 25 th and 75 th percentiles, respectively. The upper whisker is highest value within $1.5 \times$ interquartile range, measured from the upper quartile. The lower whisker is the lowest value within the $1.5 \times$ interquartile range, measured from the lower quartile. Values beyond this are represented as outlier dots.

controlled trials of cochlear implants (Chung et al., 2012; Harkonen et al., 2015a, 2015b). This might suggest that the intervention in severe-profound hearing losses would result in a greater benefit for long-term general fatigue. It is also important to distinguish the difference between hearing aid fitting and hearing aid use. Discrepancies between the amount of time hearing devices were used may account for some differences in study results.

Due to the very recent development of the VFS-A-40 scale, our finding of a significant impact of hearing aid fitting on VFS-A-40 scores at both the group and individual levels stands alone, to date. The VFS-A-40 measures listening-related fatigue, and as such seems more sensitive to the fatigue experienced by people with a hearing loss than

Table 4. Effects of Predictors on Social Activity Log Score: all Participants.

\begin{tabular}{lclc}
\hline Construct & B & SE & \multicolumn{1}{c}{$\mathrm{T}$} \\
\hline Assessment number & 2.4 & 0.79 & $3.04^{* *}$ \\
Group & 0.41 & 0.15 & $2.67^{* *}$ \\
Age (GMC) & 0.013 & 0.008 & 1.64 \\
Sex & -0.14 & 0.16 & -0.87 \\
Baseline ALDQ & 0.027 & 0.006 & $4.7^{* * *}$ \\
Baseline HHIE/A & -0.012 & 0.004 & $-3.04^{* *}$ \\
NFC & 0.013 & 0.006 & $2.26^{*}$ \\
Group $\times$ Assessment & -1.9 & 1.11 & -0.87 \\
\hline
\end{tabular}

Abbreviations: $B=$ unstandardized beta; $\mathrm{SE}=$ standard error; $t=$ test statistic; $G M C=$ grand mean centered; $A L D Q=$ auditory lifestyle demand questionnaire; $\mathrm{HHIE} / \mathrm{A}=$ hearing handicap inventory for the elderly/adults; $\mathrm{NFC}=$ need for cognition scale.

$* p<.05 * * p<.01 * * * p<.001$. general fatigue questionnaires, despite scores on all three fatigue questionnaires being significantly positively correlated at assessment 1 . The VFS-A-40 does not measure transient fatigue explicitly, but it does pose hypothetical situational questions. As a result, this study can offer no further insights into the distinct impacts of hearing aid fitting on transient and long-term fatigue. With the exception of the MFSI vigor subscale and the VFS-A-40 physical subscale, the scores on all of the other constituent subscales of the MFSI and the VFS-A-40 followed the same pattern as the total scales. MFSI subscale scores were not significantly different between groups across time points, whereas VFS-A-40 subscale score changes were significantly different between groups at each time point post fitting. These results could mean either that hearing aid fitting impacts all dimensions of

Table 5. Effects of Predictors on Social Activity Log Score: Intervention Group.

\begin{tabular}{|c|c|c|c|}
\hline Construct & $B$ & SE & $T$ \\
\hline Assessment number & 0.15 & 0.033 & $4.69 * * *$ \\
\hline Age (GMC) & -0.008 & 0.014 & -0.53 \\
\hline Sex & 0.015 & 0.22 & 0.07 \\
\hline Baseline ALDQ & 0.029 & 0.008 & $3.69 * * *$ \\
\hline Baseline HHIE/A & -0.02 & 0.005 & $3.85 * * *$ \\
\hline NFC & 0.01 & 0.008 & 1.3 \\
\hline
\end{tabular}

Abbreviations: $B=$ unstandardized beta; $\mathrm{SE}=$ standard error; $t=$ test statistic; $G M C=$ grand mean centered; $A L D Q=$ auditory lifestyle demand questionnaire; $\mathrm{HHIE} / \mathrm{A}$ = hearing handicap inventory for the elderly/adults; $\mathrm{NFC}=$ need for cognition scale. $* p<.05 * * p<.01 * * * p<.001$. 
listening-related fatigue similarly, or that people tend to report subjective fatigue as unidimensional, as has been previously suggested (Michielson et al., 2004). The lack of correlation between baseline hearing handicap and MFSI vigor does not support the previous finding of a correlation between HHIE and MFSI vigor (Hornsby \& Kipp, 2016). However, the significant improvement at the final two assessments in the intervention group suggests that vigor may play a role in listening-related fatigue.

Although a reduction in listening-related fatigue is clearly to be welcomed, it is unclear whether the change is of clinical significance. Median VFS-A-40 scores were negative for both groups at baseline (on a scale of $\sim 2$ to -2 ), which suggests that listening-related fatigue was not high for most participants, and therefore may not have been problematic. It is currently not possible for us to report VFS-A-40 data to establish whether the scores were normal for a population with audiometrically determined hearing loss, or how they compare to normal hearing norms. However, previous research has established that the threshold of minimally important difference for changes in health-related quality of life is approximately half a population standard deviation (Norman et al., 2003). At baseline, half a standard deviation in the VFS-A-40 score for the intervention group was 0.27. The change in mean VFS-A-40 from baseline to the final assessment was -0.47 , suggesting that the change in listening-related fatigue was indeed greater than the minimally important difference. For long-term general fatigue, FAS median scores calculated as a percentage were $22.5 \%$ in the intervention group and $27.5 \%$ in the control group at assessment 1. This is similar to reports from previous research where the adjusted median FAS scores for three separate hearing loss groups were $22.5 \%, 22.5 \%$, and $22 \%$ (Alhanbali et al., 2017). This indicates that long-term general fatigue in the participant sample was at expected levels.

In line with Alhanbali et al. (2018), hearing handicap correlated with fatigue. The HHIE/A was the only questionnaire to correlate significantly with all three fatigue questionnaires at baseline, and significantly associated with VFS-A-40 scores in the multilevel growth models. The absence of any relationship between fatigue and audiometric hearing loss reported by Alhanbali was also reflected in the current study. However, while Alhanbali et al. (2017) reported a significant relationship between FAS and EAS scores, here EAS was only significantly correlated with the VFS-A-40 score. The finding that the change in VFS, EAS, and HHIE/A scores correlated across time in the intervention group suggests that listening-related fatigue, hearing handicap, and listening effort may be impacted in similar ways by hearing aid fitting. It is possible that the change in listening effort could have driven the change in fatigue. However, no causal relationship between the changes can be ascertained here.

Previous cross-sectional studies suggest that hearing device fitting has a positive effect on social activity (Fisher et al., 2015; Sawyer et al., 2019). This was supported by our longitudinal data, which is an important finding in its own right. The multilevel growth model analysis accounted for a decrease in SAL score in the control group, which, given that such a drop has no apparent plausible cause, may have skewed results at assessments 2 and 3 . The differing time intervals for the measurement of social activity in the SAL (over the past month), and SPQ (over the past year), is reflected in the results, as significant improvements in the intervention group compared to the control group for the SAL were seen by assessment 2, whereas improvements in SPQ score were evident only at the final assessment.

Previous research has concluded that the control and enjoyment one has during social activity could affect the fatigue experienced (Oerlemans \& Bakker, 2014; Oerlemans et al., 2014; Ten Brummelhuis \& Trougakos, 2014). The significant improvement in social participation restriction (SPARQ) post-fitting highlighted a psychosocial benefit of hearing aid fitting, which could in part reflect improved enjoyment or control in social settings. No impact of hearing aid fitting on work or physical activity was evident in the current study.

\section{Strengths and Limitations}

The study recruited participants to the intervention group from people who were due to visit an audiology clinic as potential recipients of a hearing aid. As is normal, some of these ended up not being fitted with hearing aids, so they became part of the control group instead. There was no significant difference in VFS-A-40 score at any assessment between this subgroup and the control group participants recruited from the participant database. However, there was also a possibility that participants who did not receive a hearing aid could respond differently at the second assessment, either with improved scores due to relief (having been told that they did not need hearing aids), or worse scores (having been told they could not be helped at this point). While scores consistent with this behavior were displayed in some individuals, additional analysis showed that this had no impact on subgroup VFS-A-40 scores. Additionally, due to the variable nature of audiology appointments, the time from the first assessment to the second assessment was longer than three months for some individuals. While this was not a major issue, it could have distorted some analyses.

The difference between groups regarding age range occurred due to the extended period of recruitment and data collection, with some participants finishing as others began. Thus, any dropouts were problematic to match in the opposing group. Most participants had mild to moderate hearing losses, as the study required participants who were only just receiving their first hearing aid. It is possible that hearing aid fitting would result in more dramatic listening-related fatigue reductions for people with severe 
hearing losses, which this study could not fully investigate. On the other hand, this study represents a realistic sample of people who are likely to receive a first-ever hearing aid and therefore is more clinically relevant than one involving participants with more severe hearing losses.

The study measured hearing aid use to ensure that participants remained eligible for their assigned groups. It would be interesting to know if the level of hearing aid use (e.g., rarely vs. always) influences fatigue or even the change in fatigue post-fitting. However, the present study was not designed to investigate this question and therefore future studies would be needed to gain a better insight.

The longitudinal design of the study allowed the investigation of changes at the individual level across time. By doing so, many of the potential confounding variables in previous research were reduced or eradicated. As the FAS has been used in relevant studies of people with hearing loss before, it was the basis for sample size and power analysis. However, the FAS has, to the authors' knowledge, never been used in longitudinal assessment before. Despite this, the FAS has been shown to have excellent internal consistency, convergent validity, and test-retest reliability for various populations (Hendriks et al., 2018; Horisberger et al., 2019). By using multiple questionnaires to measure fatigue, as well as related variables, the results are more appropriate for understanding the ongoing processes related to the benefit of hearing aid fitting. It is important to mention that the version of the VFS-A-40 used in this study was a pre-publication version, with one item in the physical subscale having since changed. This item asked the respondent about taking a break from their hearing aid which is not relevant for non-hearing aid wearers. It is possible that this may have influenced physical subscale results in this study. This item was removed from the published VFS-A-40 and is therefore no longer an issue.

\section{Conclusions}

The present study is the first to use longitudinal methodology to measure fatigue before and after first-ever hearing aid fitting. It is also the first study to demonstrate a longitudinal increase in social activity level after hearing aid fitting. Hearing aid fitting showed no effect on long-term general fatigue, but there was an improvement from before fitting to six months post-fitting for listening-related fatigue. This provides a more detailed result compared to the mixed outcomes from previous research. Like social activity level, social participation restrictions improved post fitting. While the design of this study does not allow an assessment of a direct impact of social activity on fatigue, the increase in social activity and reduction in listening-related fatigue after hearing aid fitting are important findings for clinical practice as more focus should be given to extra-auditory needs and outcomes during hearing healthcare. Future research should assess whether current fatigue scales measure what we want to know in relation to hearing loss and hearing aid fitting. Research should also further investigate the impact of hearing aid use on social activity level, as well as on experiential aspects of social activity such as restriction.

\section{Acknowledgments}

The authors thank all participants who took part in the study as well as NHS Greater Glasgow \& Clyde Audiology for their help in recruitment. No potential conflict of interest was reported by the authors. This work was supported by the Medical Research Council [grant nos. MR/R502169/1, MR/S003576/1]; and by the Chief Scientist Office of the Scottish Government. The funder had no role in study design.

\section{Declaration of Conflicting Interests}

The authors declared no potential conflicts of interest with respect to the research, authorship, and/or publication of this article.

\section{Funding}

The authors disclosed receipt of the following financial support for the research, authorship, and/or publication of this article: This work was supported by the Medical Research Council [grant nos. MR/ R502169/1, MR/S003576/1]; and by the Chief Scientist Office of the Scottish Government.

\section{ORCID iDs}

Jack A. Holman iD https://orcid.org/0000-0002-9090-4809

Graham Naylor (iD https://orcid.org/0000-0003-1544-1944

\section{Supplemental Material}

Supplemental material for this article is available online.

\section{References}

Alhanbali S., Dawes P., Lloyd S., \& Munro K. J. (2017). Self-reported listening-related effort and fatigue in hearing-impaired adults. Ear and Hearing, 38(1), e39-e48. https://doi.org/10.1097/aud.0000000000000361

Alhanbali S., Dawes P., Lloyd S., \& Munro K. J. (2018). Hearing handicap and speech recognition correlate with self-reported listening effort and fatigue. Ear Hear., 39(3), 470-474. https:doi. org/10.1097/AUD.0000000000000515

Bisgaard N., \& Ruf S. (2017). Findings from EuroTrak surveys from 2009 to 2015: Hearing loss prevalence, hearing aid adoption, and benefits of hearing aid use. American Journal of Audiology, 26(3), 451-461. https://doi.org/10.1044/2017_aja16-0135

Bowman A., \& Azzalini A. (2018). Smoothing Methods for Nonparametric Regression and Density Estimation.

Burke L. A., \& Naylor G. (2020). Daily-life fatigue in mild to moderate hearing impairment: An ecological momentary assessment study. Ear and Hearing, 41(6), 1518-1532. https://doi.org/10. 1097/AUD.0000000000000888

Cacioppo J. T., \& Petty R. E. (1982). The need for cognition. Journal of Personality and Social Psychology, 42(1), 116. https://doi.org/10.1037/0022-3514.42.1.116 
Chung J., Chueng K., Shipp D., Friesen L., Chen J. M., Nedzelski J. M., \& Lin V. Y. (2012). Unilateral multi-channel cochlear implantation results in significant improvement in quality of life. Otology \& Neurotology, 33(4), 566-571. https://doi.org/ 10.1097/MAO.0b013e3182536dc2

Craig C. L., Marshall A. L., Sjöström M., Bauman A. E., Booth M. L., Ainsworth B. E., \& Sallis J. F. (2003). International physical activity questionnaire: 12-country reliability and validity. Medicine \& Science in Sports \& Exercise, 35(8), 1381-1395. https://doi.org/10.1249/01.MSS.0000078924.61453.FB

Davis H., Schlundt D., Bonnet K., Camarata S., Bess F. H., \& Hornsby B. (2021). Understanding listening-related fatigue: Perspectives of adults with hearing loss. International Journal of Audiology, 60(6), 458-468. https://doi.org/10.1080/ 14992027.2020.1834631

Dawes P., Maslin M., \& Munro K. J. (2014). 'Getting used to' hearing aids from the perspective of adult hearing-aid users. International Journal of Audiology, 53(12), 861-870. https:// doi.org/10.3109/14992027.2014.938782

Densley K., Davidson S., \& Gunn J. M. (2013). Evaluation of the Social Participation Questionnaire in adult patients with depressive symptoms using Rasch analysis. Quality of Life Research, 22(8), 1987-1997. https://doi.org/10.1007/s11136013-0354-4

Fisher D. E., Li C. M., Hoffman H. J., Chiu M. S., Themann C. L., Petersen H., \& Cotch M. F. (2015). Sex-specific predictors of hearing-aid use in older persons: The age, gene/environment susceptibility-Reykjavik study. International Journal of Audiology, 54(9), 634-641. https://doi.org/10.3109/14992027. 2015.1024889

Flensner G., Landtblom A.-M., Söderhamn O., \& Ek A.-C. (2013). Work capacity and health-related quality of life among individuals with multiple sclerosis reduced by fatigue: A cross-sectional study. BMC Public Health, 13(1), 224. https://doi.org/10.1186/ 1471-2458-13-224

Gatehouse S., Elberling C., \& Naylor G. (1999). Aspects of auditory ecology and psychoacoustic function as determinants of benefits from and candidature for non-linear processing in hearing aids. Paper presented at the Proc. 18th Danavox Symposium: Auditory models and non-linear hearing instruments.

Harkonen K., Kivekas I., Rautiainen M., Kotti V., Sivonen V., \& Vasama J. P. (2015a). Sequential bilateral cochlear implantation improves working performance, quality of life, and quality of hearing. Acta Oto-Laryngologica, 135(5), 440-446. https://doi. org/10.3109/00016489.2014.990056

Harkonen K., Kivekas I., Rautiainen M., Kotti V., Sivonen V., \& Vasama J. P. (2015b). Single-sided deafness: The effect of cochlear implantation on quality of life, quality of hearing, and working performance. $\mathrm{Orl}, 77(6), 339-345$. https://doi.org/10. 1159/000439176

Heffernan E., Coulson N. S., \& Ferguson M. A. (2018). Development of the Social Participation Restrictions Questionnaire (SPaRQ) through consultation with adults with hearing loss, researchers, and clinicians: A content evaluation study. International Journal of Audiology, 57(10), 791-799. https://doi.org/10.1080/14992027.2018.1483585

Heffernan E., Coulson N. S., Henshaw H., Barry J. G., \& Ferguson M. A. (2016). Understanding the psychosocial experiences of adults with mild-moderate hearing loss: An application of Leventhal's self-regulatory model. International Journal of
Audiology, 55(Suppl 3), S3-S12. https://doi.org/10.3109/ 14992027.2015.1117663

Heffernan E., Maidment D. W., Barry J. G., \& Ferguson M. A. (2019). Refinement and validation of the Social Participation Restrictions Questionnaire: An application of Rasch analysis and traditional psychometric analysis techniques. Ear and Hearing, 40(2), 328-339. https://doi.org/10.1097/AUD.00000 00000000618

Hendriks C., Drent M., Elfferich M., \& De Vries J. (2018). The fatigue assessment scale: Quality and availability in sarcoidosis and other diseases. Current Opinion in Pulmonary Medicine, 24(5), 495-503. https://doi.org/10.1097/MCP.0000000000000496

Hockey R. (2013). The psychology of fatigue: Work, effort and control. Cambridge University Press.

Holman J. A., Drummond A., Hughes S. E., \& Naylor G. (2019). Hearing impairment and daily-life fatigue: A qualitative study. International Journal of Audiology, 58(7), 408-416. https:// doi.org/10.1080/14992027.2019.1597284

Holman J. A., Drummond A., \& Naylor G. (2021a). The effect of hearing loss and hearing device fitting on fatigue in adults: A systematic review. Ear and Hearing, 42(1), 1-11. https://doi. org/10.1097/AUD.0000000000000909

Holman J. A., Hornsby B. W. Y., Bess F. H., \& Naylor G. (2021b). Can listening-related fatigue influence well-being? Examining associations between hearing loss, fatigue, activity levels and well-being. International Journal of Audiology, 60(sup2), 4759. https://doi.org/10.1080/14992027.2020.1853261

Horisberger A., Courvoisier D., \& Ribi C. (2019). The fatigue assessment scale as a simple and reliable tool in systemic lupus erythematosus: A cross-sectional study. Arthritis Research \& Therapy, 21(1), 80. https://doi.org/10.1186/ s13075-019-1864-4

Hornsby B. W. (2013). The effects of hearing aid use on listening effort and mental fatigue associated with sustained speech processing demands. Ear and Hearing, 34(5), 523-534. https:// doi.org/10.1097/AUD.0b013e31828003d8

Hornsby B. W., Camarata S., Cho S.-J., Davis H., McGarrigle R., \& Bess F. H. (2021). Development and validation of the Vanderbilt Fatigue Scale for Adults (VFS-A). Psychological Assessment, 33(8), 777-788. https://doi.org/10.1037/pas0001021

Hornsby B. W., \& Kipp A. M. (2016). Subjective ratings of fatigue and vigor in adults with hearing loss are driven by perceived hearing difficulties not degree of hearing loss. Ear Hear., 37(1), e1-10. https://doi.org/10.1097/AUD.0000000000000203

Hornsby B. W. Y., Naylor G., \& Bess F. H. (2016). A taxonomy of fatigue concepts and their relation to hearing loss. Ear and Hearing, 37, 136S-144S. https://doi.org/10.1097/aud. 0000000000000289

Lee D. H., \& Noh H. (2015). Prediction of the use of conventional hearing aids in Korean adults with unilateral hearing impairment. International Journal of Audiology, 54(9), 613-619. https://doi. org/10.3109/14992027.2015.1023902

Michielsen H. J., De Vries J., \& Van Heck G. L. (2003). Psychometric qualities of a brief self-rated fatigue measure: The fatigue assessment scale. Journal of Psychosomatic Research, 54(4), 345-352. https://doi.org/10.1016/s0022-3999(02)00392-6

Michielsen H. J., De Vries J., Van Heck G. L., Van de Vijver F. J., \& Sijtsma K. (2004). Examination of the dimensionality of fatigue. European Journal of Psychological Assessment, 20(1), 39-48. https://doi.org/10.1027/1015-5759.20.1.39 
Munro K. J. (2008). Reorganization of the adult auditory system: Perceptual and physiological evidence from monaural fitting of hearing aids. Trends in Amplification, 12(3), 254-271. https:// doi.org/10.1177/1084713808323483

Munro K. J., \& Lutman M. E. (2003). The effect of speech presentation level on measurement of auditory acclimatization to amplified speech. Journal of the Acoustical Society of America, 114(1), 484-495. https://doi.org/10.1121/1.1577556

Newman C. W., Weinstein B. E., Jacobson G. P., \& Hug G. A. (1990). The hearing handicap inventory for adults: Psychometric adequacy and audiometric correlates. Ear and Hearing, 11(6), 430-433. https://doi.org/10.1097/00003446199012000-00004

Norman G. R., Sloan J. A., \& Wyrwich K. W. (2003). Interpretation of changes in health-related quality of life: The remarkable universality of half a standard deviation. Medical Care, 41(5), 582592. https://doi.org/10.1097/01.MLR.0000062554.74615.4C

Oerlemans W. G. M., \& Bakker A. B. (2014). Burnout and daily recovery: A day reconstruction study. Journal of Occupational Health Psychology, 19(3), 303-314. https:/doi.org/10.1037/a0036904

Oerlemans W. G. M., Bakker A. B., \& Demerouti E. (2014). How feeling happy during off-job activities helps successful recovery from work: A day reconstruction study. Work and Stress, 28(2), 198-216. https://doi.org/10.1080/02678373.2014.901993

Pinheiro J., Bates D., DebRoy S., \& Sarkar D. (2019). nlme: Linear and Nonlinear Mixed Effects Models.

R Core Team (2019). R: A language and environment for statistical computing. R Foundation for Statistical Computing.

Sawyer C. S., Armitage C. J., Munro K. J., Singh G., \& Dawes P. D. (2019). Correlates of hearing aid use in UK adults: Self-reported hearing difficulties, social participation, living situation, health, and demographics. Ear and Hearing, 40(5), 1061-1068. https://doi.org/10.1097/aud.0000000000000695
Smith B. N., Kerr N. A., Markus M. J., \& Stasson M. F. (2001). Individual differences in social loafing: Need for cognition as a motivator in collective performance. Group Dynamics: Theory, Research, and Practice, 5(2), 150. https://doi.org/10. 1037/1089-2699.5.2.150

Stein K.D., Jacobsen P.B., Blanchard C.M., \& Thors C. (2004). Further validation of the multidimensional fatigue symptom inventory-short form. J Pain Symptom Manage., 27(1), 14 23. https://doi.org/10.1016/j.jpainsymman.2003.06.003

Syrjala K. L., Stover A. C., Yi J. C., Artherholt S. B., \& Abrams J. R. (2010). Measuring social activities and social function in long-term cancer survivors who received hematopoietic stem cell transplantation. Psycho-Oncology, 19(5), 462-471. https:// doi.org/10.1002/pon.1572

Ten Brummelhuis L. L., \& Trougakos J. P. (2014). The recovery potential of intrinsically versus extrinsically motivated off-job activities. Journal of Occupational and Organizational Psychology, 87(1), 177-199. https://doi.org/10.1111/joop.12050

Ventry I. M., \& Weinstein B. E. (1982). The hearing handicap inventory for the elderly: A new tool. Ear and Hearing, 3(3), 128-134. https://doi.org/10.1097/00003446-198205000-00006

Vestergaard Knudsen L., Öberg M., Nielsen C., Naylor G., \& Kramer S. E. (2010). Factors influencing help seeking, hearing aid uptake, hearing aid use and satisfaction with hearing aids: A review of the literature. Trends in Amplification, 14(3), 127154. https://doi.org/10.1177/1084713810385712

Vigour N. (2020). Oxford English dictionary. Oxford: Oxford University Press.

Whiteneck G. G., Charlifue S. W., Gerhart K. A., Overholser J. D., \& Richardson G. N. (1992). Quantifying handicap: A new measure of long-term rehabilitation outcomes. Archives of Physical Medicine and Rehabilitation, 73(6), 519-526. https:// doi.org/10.5555/uri:pii:000399939290185Y 